\title{
Project VeSElkA : Preliminary results for CP stars recently observed with ESPaDOnS $\dagger$
}

\author{
Viktor Khalack and Francis LeBlanc \\ Université de Moncton, Moncton, Canada \\ email: khalakv@umoncton.ca
}

\begin{abstract}
We present the first results for the estimation of gravity and effective temperature of poorly studied chemically peculiar stars recently observed with the spectropolarimeter ESPaDOnS at CFHT in the frame of the VeSElkA (Vertical Stratification of Elements Abundance) project. A grid of theoretical stellar atmosphere models with the corresponding fluxes has been calculated using the PHOENIX code. We have used these fluxes to fit Balmer line profiles employing the code FITSB2 that produces estimates of the effective temperature, surface gravity and radial velocity for each star.
\end{abstract}

Keywords. stars: atmospheres, stars: chemically peculiar, stars: fundamental parameters

\section{Introduction}

Detection of vertical abundance stratification of chemical species in atmospheres of chemically peculiar (CP) stars is considered to be an indicator of the effectiveness of the diffusion mechanism in their atmospheres. It is also responsible for the observed peculiarities of chemical abundances. Therefore, we have initiated a new project entitled "Vertical Stratification of Elements Abundance" (VeSElkA - meaning rainbow in Ukrainian) aimed to search for and study the signatures of abundance stratification of chemical species with optical depth in the atmospheres of slowly rotating CP stars. A list of relatively bright and poorly studied CP stars that may be observed with ESPaDOnS has been compiled based on the catalog of CP stars of Renson \& Manfroid (2009). In stars without surface convection, their slow rotation supports the hypothesis of a hydrodynamically stable atmosphere. This results in comparatively narrow and unblended line profiles that are suitable for abundance analysis. High resolution $(\mathrm{R}=65000)$ Stokes IV spectra of several CP stars were recently obtained with ESPaDOnS in the spectral domain from $3700 \AA$ to $10000 \AA$ with maximal $\mathrm{S} / \mathrm{N} \simeq 1000$.

\section{Grid of models}

A new library of high resolution synthetic spectra has been created in order to determine $T_{\text {eff }}$ and $\log g$ of stars observed in the frame of the VeSElkA project. A grid of stellar atmosphere models and corresponding fluxes with high spectral resolution $(\mathrm{R}=60000)$ have been calculated using version 15 of the PHOENIX code (Hauschildt et al. 1997) for: $5000 \mathrm{~K}<T_{\text {eff }}<9000 \mathrm{~K}$ with a $250 \mathrm{~K}$ step, $9000 \mathrm{~K}<T_{\text {eff }}<15000 \mathrm{~K}$ with a $500 \mathrm{~K}$ step, $3.5<\log g<4.5$ with a 0.5 step, and $[\mathrm{Fe} / \mathrm{H}]=-1.0,-0.5,0.0,+0.5,+1.0,+1.5$.

$\dagger$ Based on observations obtained at the Canada-France-Hawaii Telescope (CFHT) which is operated by the National Research Council of Canada, the Institut National des Sciences de l'Univers of the Centre National de la Recherche Scientifique of France, and the University of Hawaii. 
Table 1. List of the observed slowly rotating CP stars.

\begin{tabular}{l|rrrrcrc|c}
\hline Star & $\boldsymbol{m}_{\mathbf{V}}$ & $\boldsymbol{\Delta} \boldsymbol{t}(\mathbf{s})$ & $\mathbf{S} / \mathbf{N}$ & $\boldsymbol{T}_{\text {eff }}(\mathbf{K})$ & $\log \boldsymbol{g}$ & $\boldsymbol{V}_{\mathbf{r}}(\mathbf{k m} / \mathbf{s})$ & $\chi^{\mathbf{2}} / \boldsymbol{\nu}$ & $\boldsymbol{V} \sin \boldsymbol{i}(\mathbf{k m} / \mathbf{s})$ \\
\hline HD 15385 & 6.2 & 1600 & 1100 & $8230 \pm 200$ & $4.00 \pm 0.2$ & $22.0 \pm 1.0$ & 0.65 & 29.0 \\
HD 22920 & 5.5 & 920 & 1150 & $13640 \pm 200$ & $3.72 \pm 0.2$ & $15.7 \pm 2.3$ & 2.08 & $40.0 \pm 2.1$ \\
HD 23878 & 5.2 & 660 & 950 & $8740 \pm 200$ & $3.86 \pm 0.2$ & $29.5 \pm 1.0$ & 0.62 & 24.0 \\
HD 68351 & 5.6 & 920 & 1100 & $10050 \pm 200$ & $3.22 \pm 0.2$ & $18.1 \pm 2.0$ & 1.17 & 33.0 \\
HD 71030 & 6.1 & 1140 & 1100 & $6780 \pm 200$ & $4.04 \pm 0.2$ & $38.1 \pm 1.0$ & 0.28 & $9.0 \pm 2.0$ \\
HD 83373 & 6.4 & 1524 & 1000 & $9800 \pm 200$ & $3.81 \pm 0.2$ & $26.5 \pm 1.0$ & 0.92 & 28.0 \\
HD 90277 & 4.7 & 520 & 1000 & $7250 \pm 200$ & $3.62 \pm 0.2$ & $14.5 \pm 1.0$ & 1.29 & 34.0 \\
HD 95608 & 4.4 & 416 & 1300 & $9200 \pm 200$ & $4.25 \pm 0.2$ & $-10.4 \pm 1.0$ & 0.59 & $17.0 \pm 2.0$ \\
HD 97633 & 3.3 & 152 & 1300 & $8750 \pm 200$ & $3.45 \pm 0.2$ & $8.2 \pm 1.0$ & 0.61 & 23.0 \\
HD 110380 & 3.6 & 200 & 1300 & $6980 \pm 200$ & $4.19 \pm 0.2$ & $-17.6 \pm 1.0$ & 0.31 & 23.0 \\
HD 116235 & 5.9 & 1040 & 870 & $8900 \pm 200$ & $4.33 \pm 0.2$ & $-10.3 \pm 1.0$ & 0.49 & $20.0 \pm 2.0$ \\
HD 164584 & 5.4 & 880 & 1300 & $6800 \pm 200$ & $3.54 \pm 0.2$ & $-11.2 \pm 1.0$ & 1.16 & 30.0 \\
HD 186568 & 6.0 & 1300 & 1000 & $11070 \pm 200$ & $3.44 \pm 0.2$ & $-9.5 \pm 1.0$ & 1.81 & 15.0 \\
HD 209459 & 5.8 & 1160 & 1000 & $10620 \pm 200$ & $3.73 \pm 0.2$ & $-0.3 \pm 1.0$ & 1.31 & 4.0 \\
HD 223640 & 5.2 & 680 & 1200 & $12250 \pm 200$ & $3.94 \pm 0.2$ & $17.0 \pm 2.0$ & 2.04 & 28.0 \\
\hline
\end{tabular}

To verify the accuracy of the grids of stellar atmosphere models, we have used a spectrum of Vega obtained with ESPaDOnS in the spectropolarimetric mode. The profiles of nine Balmer lines in the non-normalised spectrum of Vega have been fitted with the help of the FITSB2 code (Napiwotzki et al. 2004) using the grid of models calculated for metallicity $[\mathrm{Fe} / \mathrm{H}]=-0.5$. The best fit is obtained for $T_{\text {eff }}=9690 \mathrm{~K}$ and $\log g=4.18$, resulting in $\chi^{2} / \nu=2.01$. Exactly the same approach has been applied to fit nine Balmer lines in the same spectrum using the grid of models calculated with version 16 of the PHOENIX code for the metallicity $[\mathrm{Fe} / \mathrm{H}]=-0.5$ (see Husser et al. (2013) for more detail). The best fit has resulted in $T_{\text {eff }}=9630 \mathrm{~K}$ and $\log g=4.14$ with $\chi^{2} / \nu=2.00$. These values of effective temperature and surface gravity are close to one another and to the values $T_{\text {eff }}=9560 \mathrm{~K}$ and $\log g=4.05$ obtained for Vega by Hill \& Landstreet (1993) using the results of $u b v y H$ photometry.

\section{Stellar atmosphere parameters for program stars}

Nine Balmer line profiles have been fitted in the observed spectra of the selected slowly rotating CP stars to find their effective temperature, surface gravity and radial velocity (see Table 1). The fitting procedure has been performed with the help of FITSB2 code (Napiwotzki et al. 2004), employing our grids of stellar atmosphere models (calculated with PHOENIX 15) together with the respective simulated spectra. For each star, the fitting procedure has been performed for the metallicities $[\mathrm{Fe} / \mathrm{H}]=-1.0,-0.5,0.0,+0.5$, +1.0. Among the obtained results, the fundamental parameters corresponding to the fit with the smallest value $\chi^{2} / \nu$ are chosen. Our final results are consistent with the previously published results for ten of the stars presented in Tab. 1, and we have determined $T_{\text {eff }}$ and $\log g$ for five other CP stars (HD 23878, HD 68351, HD 83373, HD 164584 and HD 186568) for the first time.

\section{References}

Hauschildt, P. H., Shore, S. N., Schwarz, G. J., et al. 1997, ApJ 490, 803

Hill, G. M. \& Landstreet, J. D. 1993, A\&A 276, 142

Husser, T.-O., Wende-von Berg, S., Dreizler, S., et al. 2013, A\&A 553, 6

Napiwotzki, R., Yungelson, L., Nelemans, G., et al. 2004, ASPC 318, 402

Renson, P. \& Manfroid, J. 2009, A\&SA 498, 961 